\title{
Rosiglitazone exerts neuroprotective effects via the suppression of neuronal autophagy and apoptosis in the cortex following traumatic brain injury
}

\author{
JUNCHAO YAO $^{1}$, KEBIN ZHENG $^{2}$ and XIANG ZHANG ${ }^{1}$ \\ ${ }^{1}$ Department of Neurosurgery, Central Hospital of Cangzhou, Cangzhou, Hebei 061000; \\ ${ }^{2}$ Department of Neurosurgery, Affiliated Hospital of Hebei University, Baoding, Hebei 071000, P.R. China
}

Received September 29, 2014; Accepted June 17, 2015

DOI: $10.3892 / \mathrm{mmr} .2015 .4292$

\begin{abstract}
Traumatic brain injury (TBI) is one of the leading causes of mortality and morbidity in adults and children worldwide. Recent studies have demonstrated that both apoptosis and autophagy participate in TBI-induced neuronal cell death and functional loss. The peroxisome proliferator-activated receptor- $\gamma$ (PPAR- $\gamma$ ) agonist rosiglitazone (RSG) is a well-known anti-inflammatory, which carries out its effects via the activation of PPAR- $\gamma$. Previous studies have suggested that RSG may exert neuroprotective effects in animal models of both chronic and acute brain injury; however, whether RSG is involved in autophagic neuronal death following TBI remains to be elucidated. The present study aimed to determine whether RSG carries out its neuroprotective properties via the attenuation of neuronal apoptosis and autophagy, following TBI in a rat model. Furthermore, the role of RSG was investigated with regards to the modulation of inflammation and glutamate excitotoxicity, and the impact of RSG on functional recovery following TBI was determined. The rats were subjected to controlled cortical impact injury, prior to being randomly divided into three groups: A sham-operated group, a TBI group, and an RSG treatment group. The RSG treatment group was intraperitoneally treated with $2 \mathrm{mg} / \mathrm{kg}$ RSG immediately after TBI. The results of the present study demonstrated that RSG treatment following TBI significantly reduced neuronal apoptosis and autophagy, and increased functional recovery. These effects were correlated with a decrease in the protein expression levels of tumor necrosis factor $\alpha$ and interleukin-6. However, no significant changes were observed in the protein expression levels of glutamate transporter-1 in the brain cortex. The results of the present study provide in vivo evidence that
\end{abstract}

Correspondence to: Dr Junchao Yao, Department of Neurosurgery, Central Hospital of Cangzhou, 16 Xinhua Western Road, Cangzhou, Hebei 061000, P.R. China

E-mail: junchaoyao24k@163.com

Key words: rosiglitazone, neuroprotection, traumatic brain injury, inflammatory, autophagy, apoptosis, rats
RSG may exert neuroprotective effects via the inhibition of neuronal apoptosis and autophagy following experimental TBI in rats, and the mechanism underlying these effects may be associated with the anti-inflammatory action of RSG. The present study offers a novel insight into the potential use of RSG as a neuroprotective agent for the treatment of cerebral injuries.

\section{Introduction}

Traumatic brain injury (TBI) is one of the leading causes of mortality and morbidity in adults and children worldwide (1). Tissue loss and cell death following TBI results from both primary injury (direct physical tissue disruption) and secondary injury (delayed molecular pathophysiological changes) (2). Secondary injury occurs following primary injury and may continue for days or weeks, resulting in progressive neuronal death $(2,3)$. The mechanisms underlying secondary injury include inflammation, glutamate excitotoxicity, neuronal death and neurological dysfunction, all of which result in the induction of mitochondrial dysfunction as well as the amplification of biochemical cell death signaling cascades (4-6).

A recent study confirmed that both apoptosis and autophagy participate in TBI-induced neuronal cell death and functional loss (7). In addition, neuronal apoptosis has a significant role in the pathophysiology of TBI (8). Furthermore, caspase-3 and $\mathrm{B}$ cell lymphoma 2 (Bcl-2) are recognized as important apoptotic regulators, and the expression levels of caspase- 3 and Bcl-2 determine the fate of cells $(9,10)$. A previous study demonstrated that TBI-activated autophagy and increased microtubule-associated protein 1 light chain 3 (LC3) immunostaining occurred predominantly in neurons (11). Beclin-1 has also been shown to participate in the regulation of neuronal autophagy (12). These results indicate that numerous cell apoptosis mechanisms may contribute to TBI-induced neuronal cell death. Therefore, identifying neuroprotective agents that inhibit these numerous cell death mechanisms may provide novel therapeutic strategies for the treatment of TBI.

Rosiglitazone (RSG) is a peroxisome proliferator-activated receptor- $\gamma$ (PPAR- $\gamma$ ) agonist, known for its anti-inflammatory actions via PPAR- $\gamma$ activation (13). Previous studies have suggested that RSG may exert neuroprotective effects in 
animal models of chronic brain injuries, such as Alzheimer's disease (14), amyotrophic lateral sclerosis (15), and Parkinson's disease (16). The efficacy of RSG has also been demonstrated in animal models of acute brain injuries, including focal ischemia (17), spinal cord injury (18), and TBI (19). A recent study suggested that treatment with RSG attenuated TBI-induced excessive neuronal apoptosis (19); however, whether RSG treatment is involved in TBI-induced autophagic neuronal death remains unclear.

In order to determine the potential mechanism underlying the neuroprotective effects of RSG following TBI, the present study aimed to investigate the hypothesis that RSG carries out its neuroprotective effects via the attenuation of neuronal apoptosis and autophagy following TBI in rats. Furthermore, the role of RSG in the modulation of inflammatory and glutamate excitotoxicity, and the impact of RSG on the progression of functional recovery following TBI were also investigated.

\section{Materials and methods}

Animals. Adult female Sprague Dawley rats (weight, 250-300 g; age, 3 months; Hebei University Animal Center, Baoding, China) were used for the experiments of the present study. All experiments were performed in acordance with the institutional guidelines for the care and use of laboratory animals (Hebei University School of Medicine). All rats were provided with ad libitum access to food and water prior to experimentation, and were housed in a $12 \mathrm{~h}$ light/dark environment at $22^{\circ} \mathrm{C}$.

Models of TBI. Controlled cortical impact (CCI) injury was carried out on the rats as previously described (1). The rats were anesthetized by intraperitoneal injection of $50 \mathrm{mg} / \mathrm{kg}$ sodium pentobarbital (Beijing Solarbio Science \& Technology Co., Ltd., Beijing, China), prior to being placed in a stereotaxic frame. A $5 \mathrm{~mm}$ craniotomy was performed over the left parietal cortex, centered on the coronal suture and $3 \mathrm{~mm}$ laterally to the sagittal suture. Considerable care was taken to avoid injury to the underlying dura. CCI was performed using a pneumatic piston with a rounded metal tip ( $2.5 \mathrm{~mm}$ diameter), angled $22.5^{\circ}$ from vertical so that the metal tip was perpendicular to the brain surface at the center of the craniotomy. A velocity of $4 \mathrm{~m} / \mathrm{s}$ and a deformation depth of $2 \mathrm{~mm}$ below the dura were used. The bone flap was immediately replaced and sealed, and the scalp was closed with sutures (Beijing Solarbio Science \& Technology Co., Ltd.). Body temperature was monitored throughout the surgery using a rectal probe, and the temperature was maintained at $37.0 \pm 0.5^{\circ} \mathrm{C}$ using a heated pad. The rats were subsequently placed in a heated cage in order to maintain constant body temperature while recovering from anesthesia.

Groups and drug administration. The rats were randomly assigned to a sham-operated group (sham, $n=30$ ); a TBI group, which received $0.9 \%$ saline solution (vehicle, $n=60$ ); and a TBI group, which was treated with RSG (RSG, n=60; Cell Signaling Technology, Inc., Danvers, MA, USA). RSG was dissolved in $0.9 \%$ saline and stored at $4{ }^{\circ} \mathrm{C}$. A total of $2 \mathrm{mg} / \mathrm{kg}$ RSG was administered via intraperitoneal injection in the RSG group immediately after TBI. All experiments were carried out as blind studies, and the animal codes were only revealed at the end of the behavioral and histologic analyses.

Immunofluorescence. The rats were sacrificed $24 \mathrm{~h}$ after TBI by exsanguination. Prior to exsanguination, the rats were anesthetized with sodium pentobarbital (i.p.;50 mg/kg). Coronal sections $(10 \mu \mathrm{m})$ were obtained from the anterior area of the left hemisphere. The sections were incubated with $10 \%$ normal donkey serum (Beijing Solarbio Science \& Technology Co., Ltd.) for $30 \mathrm{~min}$ at room temperature in phosphate-buffered saline (PBS) supplemented with 0.1\% Triton X-100 (Beijing Solarbio Science \& Technology Co., Ltd.), prior to being incubated with the appropriate primary antibodies overnight at $4^{\circ} \mathrm{C}$. The following primary antibodies were used in various combinations: Anti-neuron-specific nuclear protein (NeuN) (1:200; cat. no. sc-134481) and anti-caspase 3 (1:50; cat. no. sc-98785), and anti-LC3 (1:50; cat. no. sc-54237) (Santa Cruz Biotechnology, Inc., Dallas, TX, USA). Following primary antibody incubation, the coronal sections were washed four times at room temperature, prior to being incubated with appropriate fluorescence-labeled secondary antibodies (1:200) for $1 \mathrm{~h}$ at room temperature. A total of $5 \mu \mathrm{g} / \mathrm{ml} \mathrm{4}$,6-diamidino-2-phenylindole (Beijing Solarbio Science \& Technology Co., Ltd.) was incubated with the coronal sections in order to carry out counterstaining of the nucleus. The sections were then washed with PBS and mounted onto slides using water-based mounting medium containing anti-fading agents (Thermo Fisher Scientific, Inc., Waltham, MA, USA). All confocal images were captured using an Olympus FV1000 laser scanning confocal microscope, and were analyzed using FV10-ASW 1.5 Viewer digital imaging software (Olympus Corporation, Tokyo, Japan).

Western blot analysis. Briefly, the rats were anesthetized with sodium pentobarbital (i.p.; $50 \mathrm{mg} / \mathrm{kg}$ ) and underwent intracardiac perfusion with $0.1 \mathrm{~mol} / \mathrm{l} \mathrm{PBS}$ ( $\mathrm{pH} 7.4$ ). The cortex region of the brain was rapidly isolated, homogenized (BestBio Biotechnology, Beijing, China), and total proteins were extracted using protein extraction reagent (Bio-Rad Biotechnology, Inc., Shanghai, China). Protein concentration was determined using a bicinchoninic acid assay (Beijing Solarbio Science \& Technology Co., Ltd., Beijing, China). The samples were separated by $20 \%$ SDS-PAGE, and were then transferred onto polyvinylidene fluoride membranes (Roche Diagnostics GmbH, Mannheim, Germany) prior to being blocked with $5 \%$ fat-free dry milk for $1 \mathrm{~h}$ at room temperature. The membranes were subsequently incubated with the following primary antibodies overnight at $4^{\circ} \mathrm{C}$ : Rabbit anti-tumor necrosis factor- $\alpha$ (TNF- $\left.\alpha\right)$ polyclonal antibody (cat. no. sc-7895), rabbit anti-interleukin (IL)-6 polyclonal antibody (cat. no. sc-7920), rabbit anti-glutamate transporter-1 (GLT-1) polyclonal antibody (cat. no. sc-365634), rabbit anti-capase 3 polyclonal antibody (cat. no. sc-7148), rabbit anti-Bcl-2 polyclonal antibody (cat. no. sc-783), rabbit anti-beclin polyclonal antibody (cat. no. sc-292327), rabbit anti-LC3 polyclonal antibody (cat. no. sc-134226), and mouse anti- $\beta$-actin monoclonal antibody (cat. no. sc-376421) (all 1:500; Santa Cruz Biotechnology, Inc.). The membranes were then incubated with horseradish peroxidase-conjugated anti-rabbit immunoglobulin (Ig)G (cat. no. sc-2027) and anti-mouse IgG 
(cat. no. sc-2025) (1:5,000; Cell Signaling Technology, Inc.) for $2 \mathrm{~h}$ at room temperature. The membrane was then visualized with an Enhanced Chemiluminescence Detection system (BestBio Biotechnology) and the densitometric signals were quantified using ImageJ 1.41 software (National Institutes of Health, Bethesda, MD, USA). The immunoreactive bands of the proteins were normalized to the band intensity of $\beta$-actin. The western blot results were analyzed using ImageJ 1.41 software (National Institutes of Health, Bethesda, MA, USA).

Recovery of motor function. The neurobehavioral status of the rats was evaluated using a set of 10 tasks, collectively termed the neurological severity score (NSS), which tests the reflexes, alertness, coordination, and motor abilities of the rats. One point is awarded for failure to perform a particular task, and thus a score of 10 reflects maximum impairment, whereas a score of 0 is normal. The NSS was evaluated at 1, 4 and 7 days post-injury. An observer who was unaware of the treatment the animal had received assessed each rat. The difference between the initial NSS and the NSS at a later time point was calculated for each rat $(\Delta \mathrm{NSS})$, and this value reflects the spontaneous or treatment-induced recovery of motor function.

Statistical analysis. All data were presented as the mean \pm standard error. SPSS 16.0 (SPSS, Inc., Chicago, IL, USA) was used for all statistical analyses of the data. Statistical analysis was performed using one-way analysis of variance, followed by Student-Newman-Keuls post-hoc tests. $\mathrm{P}<0.05$ was considered to indicate a statistically significant difference.

\section{Results}

Treatment with RSG attenuates TBI-induced motor deficits. Fig. 1 exhibits the temporal changes in functional recovery of the TBI rats, expressed as $\triangle$ NSS. Post-injury administration of RSG markedly improved motor function recovery on days 1,4 , and 7 following TBI.

Treatment with RSG inhibits caspase 3 expression in the cortex following TBI. Co-localization of NeuN and caspase 3 was assessed by immunofluorescent staining on day 1 . As shown in Fig. 2A, the majority of TBI-induced apoptosis occurred in the neurons. As demonstrated in Fig. 2B, 1, 4 and 7 days after TBI, the expression levels of caspase 3 were markedly increased in the TBI group, as compared with the sham group, and treatment with RSG markedly attenuated caspase 3 expression, as compared with the TBI group (Fig. 2C).

$R S G$ treatment increases the expression levels of Bcl-2 in the cortex following TBI. The protein expression levels of Bcl-2 in the cortex were analyzed by western blotting 1, 4 and 7 days after TBI (Fig. 3). As shown in Fig. 3, the expression levels of Bcl-2 were significantly downregulated in the TBI group, as compared with the sham group 1,4 and 7 days after TBI. Treatment with RSG markedly increased the expression levels of Bcl-2, as compared with the TBI group.

Treatment with RSG decreases the expression levels of LC3-II/LC3-I in the cortex following TBI. A recent study demonstrated that the expression levels of autophagy marker protein LC3 were significantly increased 1 day following TBI (7); therefore, the present study investigated the co-localization of NeuN and LC3 using immunofluorescent staining 1 day after TBI. As shown in Fig. 4A, the majority of TBI-induced autophagy occurred in the neurons. As demonstrated in Fig. 4B and C, 1, 4 and 7 days following TBI, the expression levels of LC3-II/LC3-I were significantly increased in the TBI group, as compared with the sham group, and treatment with RSG significantly decreased the expression levels of LC3-II/LC3-I in the rat cortex, as compared with the TBI group.

Treatment with RSG decreases the expression levels of Beclin-1 in the cortex following TBI. The expression levels of Beclin-1 in the cortex were measured by western blot analysis 1,4 and 7 days after TBI (Fig. 5). As shown in Fig. 5, the expression levels of Beclin-1 were significantly increased at the various time points in the TBI group, as compared with the sham group. Conversely, treatment with RSG significantly reduced the expression levels of Beclin-1, as compared with the TBI group.

Treatment with RSG decreases the expression levels of IL-6 in the cortex following TBI. The expression levels of IL-6 in the cortex were measured by western blot analysis 1, 4 and 7 days after TBI (Fig. 6). The expression levels of IL-6 were significantly increased at the various time points in the TBI group, as compared with the sham group. Conversely, treatment with RSG significantly reduced the expression levels of IL-6, as compared with the TBI group.

Treatment with RSG attenuates the expression levels of TNF- $\alpha$ in the cortex following TBI. The expression levels of TNF- $\alpha$ in the cortex were measured by western blot analysis 1,4 and 7 days after TBI (Fig. 7). The expression levels of TNF- $\alpha$ were significantly increased at the various time points in the TBI group, as compared with the sham group. Conversely, treatment with RSG significantly reduced the TBI-induced upregulation of TNF- $\alpha$ expression.

No significant changes were observed in the protein expression levels of GLT-1 in the cortex following RSG treatment. The protein expression levels of GLT-1 in the cortex were analyzed by western blotting at 1, 4 and 7 days after TBI (Fig. 8). The expression levels of GLT-1 were significantly downregulated in the TBI group, as compared with the sham group 1, 4 and 7 days after TBI; however, treatment with RSG induced no significant changes in the expression levels of GLT-1, as compared with the TBI group.

\section{Discussion}

The present study investigated the effectiveness of RSG, a PPAR $-\gamma$ agonist, as a therapeutic option for the treatment of TBI. The results indicated that a single injection of RSG immediately following TBI significantly reduced neuronal apoptosis and autophagy, and increased functional recovery. These effects correlate with a decrease in the protein expression levels of TNF- $\alpha$ and IL-6 in the brain cortex. However, no significant changes were observed in the protein expression levels of GLT-1 in the rats treated with RSG. Previous studies 


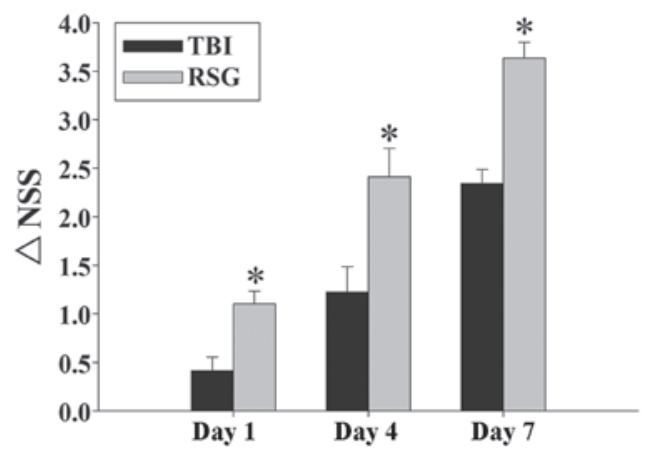

Figure 1. Effects of RSG on TBI-induced motor deficits. The temporal changes in motor recovery of the rats were determined 1, 4 and 7 days after TBI, and calculated as the difference between the initial NSS and the NSS at a later time point ( $\triangle$ NSS). The data are presented as the mean \pm standard error ( $\mathrm{n}=5$ ). Treatment with RSG significantly improved motor function 1,4 and 7 days after TBI ("P<0.01, vs. the TBI group), as reflected by an increase in $\Delta$ NSS. TBI, traumatic brain injury; RSG, rosiglitazone; NSS, neurological severity score.
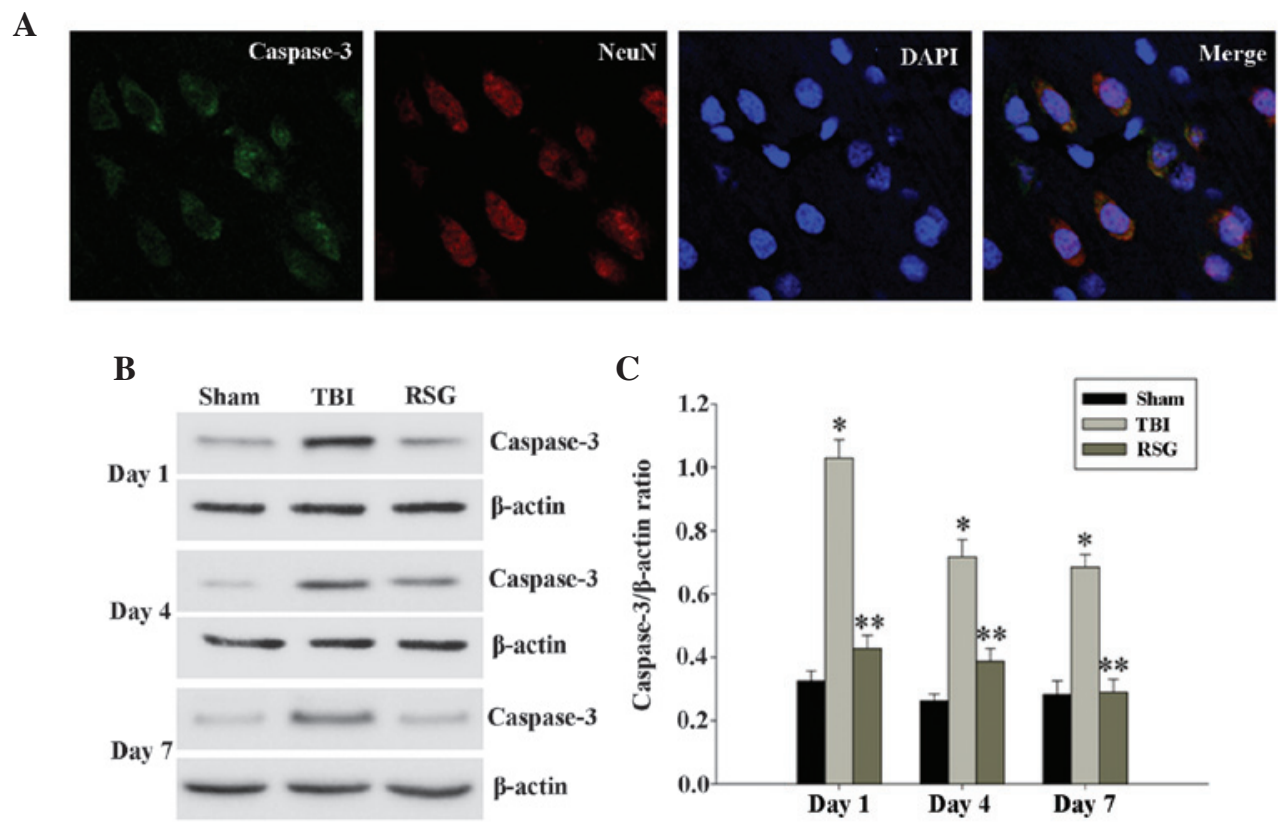

C

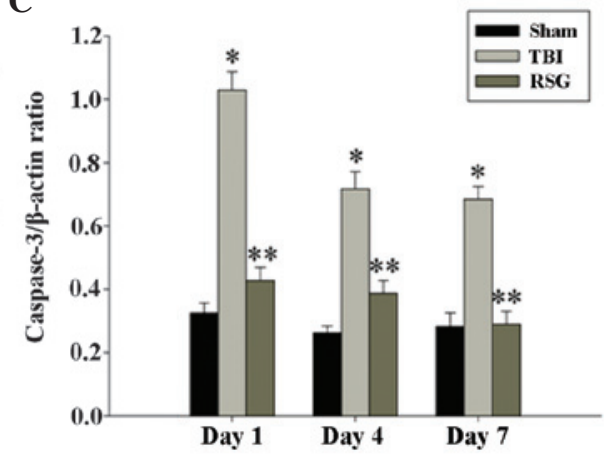

Figure 2. (A) Co-localization of NeuN and caspase $324 \mathrm{~h}$ after TBI was determined by immunofluorescent staining (magnification, $\mathrm{x} 400$ ), and cell nucleus counterstaining by 4',6-diamidino-2-phenylindole. (B) Western blot analysis was used to detect the expression levels of caspase 3 and $\beta$-actin in the cortex at 1, 4 and 7 days after TBI. (C) The expression levels of caspase 3 were normalized to those of $\beta$-actin. The data are presented as the mean \pm standard error $(\mathrm{n}=5)$. The expression levels of caspase 3 were significantly increased in the TBI group 1,4 and 7 days after TBI ("P $<0.01$, vs. the sham group), and treatment with RSG significantly decreased the protein expression levels of caspase 3 ("* $\mathrm{P}<0.05$, vs. the TBI group). TBI, traumatic brain injury; RSG, rosiglitazone; NeuN, neuron-specific nuclear protein.
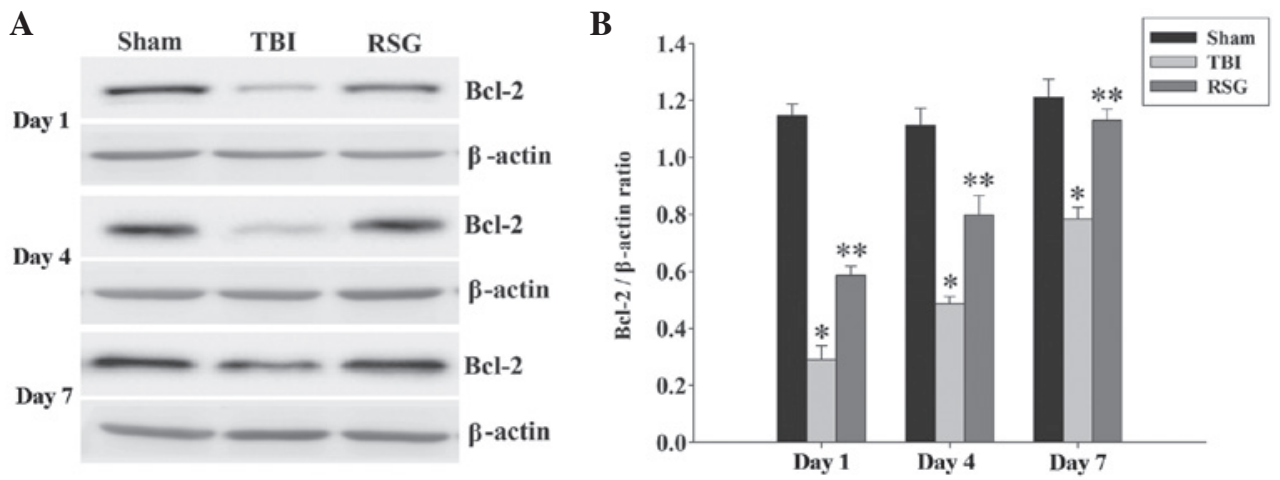

Figure 3. (A) Western blotting was used to detect the expression levels of Bcl-2 in the rat cortex 1, 4 and 7 days after TBI or sham operation. (B) The expression levels of $\mathrm{Bcl}-2$ were normalized to those of $\beta$-actin. The data are presented as the mean \pm standard error $(\mathrm{n}=5)$. The expression levels of $\mathrm{Bcl}-2$ were significantly downregulated in the TBI group 1,4 and 7 days after TBI. (" $\mathrm{P}<0.01$, vs. the sham group). Treatment with RSG significantly increased the expression levels of Bcl-2 1, 4 and 7 days after TBI $\left({ }^{* *} \mathrm{P}<0.05\right.$, vs. the TBI group). TBI, traumatic brain injury; RSG, rosiglitazone; Bcl-2, B-cell lymphoma 2. 
A
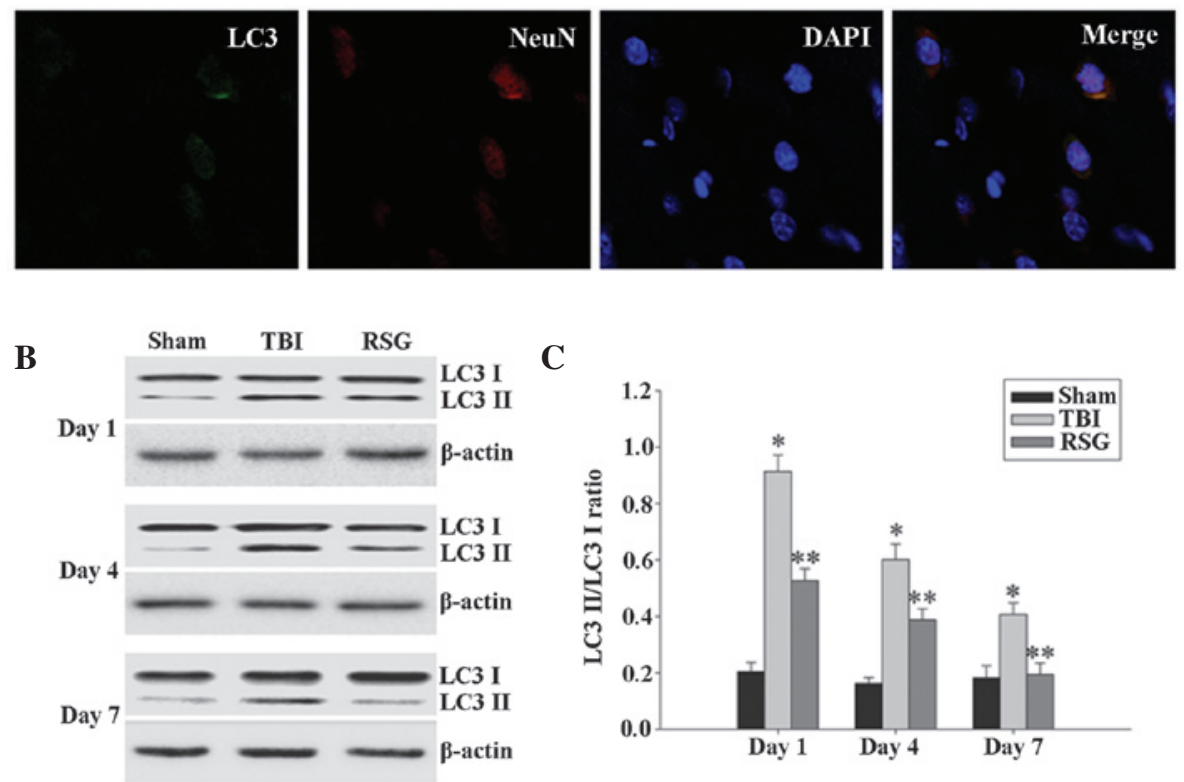

Figure 4. (A) Co-localization of NeuN and LC3 $24 \mathrm{~h}$ following TBI as determined by immunofluorescent staining (magnification, $\mathrm{x} 400$ ), and cell nucleus counterstaining by 4',6-diamidino-2-phenylindole. (B) Western blot analysis was used to detect the expression levels of LC3-II/LC3-I in the cortex 1,4 and 7 days after TBI. (C) The expression levels of LC3-II were normalized to those of LC3-I. The data are presented as the mean \pm standard error $(\mathrm{n}=5$ ). The expression levels of LC3-II/LC3-I were significantly increased in the TBI group 1, 4 and 7 days after TBI ("P $<0.01$, vs. sham group), and treatment with RSG significantly decreased the expression levels of LC3-II/LC3-I ( ${ }^{* *} \mathrm{P}<0.05$, vs. TBI group). TBI, traumatic brain injury; RSG, rosiglitazone; LC3, microtubule-associated protein 1 light chain 3; NeuN, neuron-specific nuclear protein.
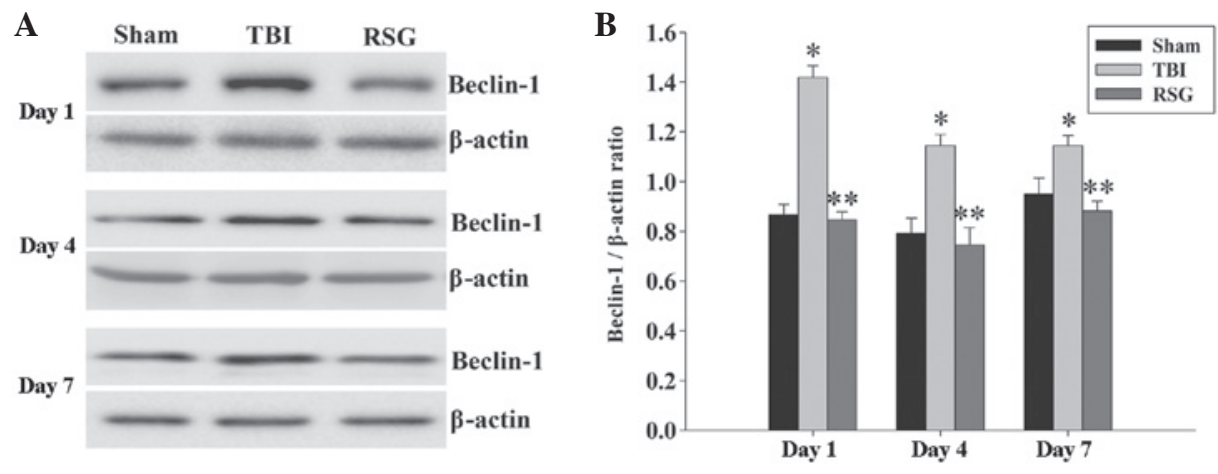

Figure 5. (A) Western blot analysis was used to detect the expression levels of Beclin-1 in the rat cortex 1, 4 and 7 days after TBI or sham operation. (B) The expression levels of Beclin-1 were normalized to those of $\beta$-actin. The data are presented as the mean \pm standard error $(n=5)$. The expression levels of Beclin-1 were significantly increased in the TBI group 1, 4 and 7 days after TBI ("P<0.01, vs. sham group). Treatment with RSG significantly downregulated Beclin-1 expression 1, 4 and 7 days after TBI (** $\mathrm{P}<0.05$, vs. TBI group). TBI, traumatic brain injury; RSG, rosiglitazone.
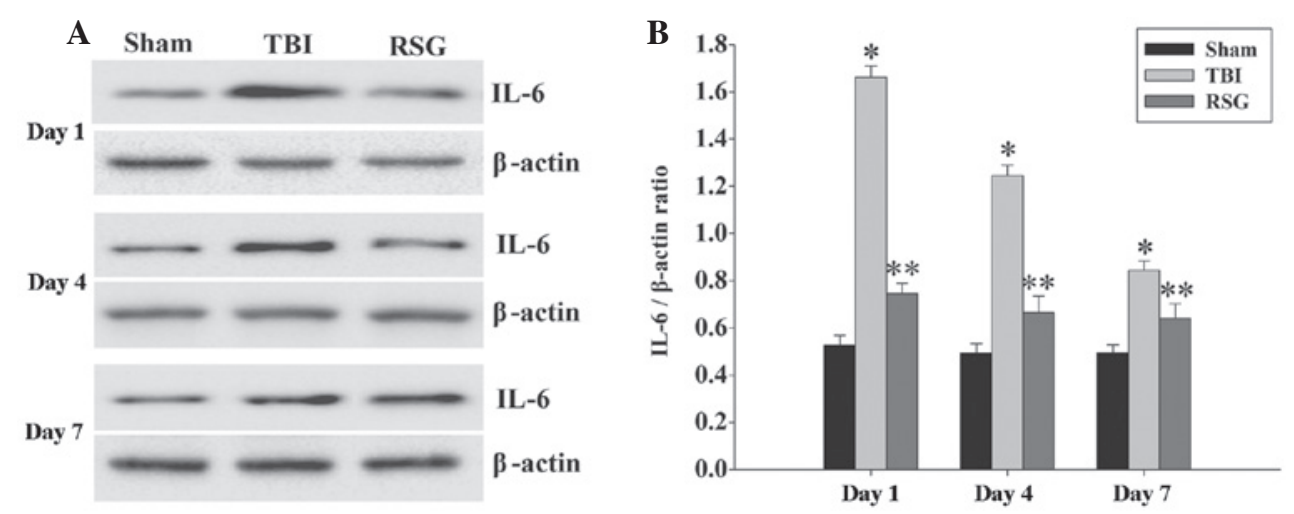

Figure 6. (A) Western blot analysis was used to detect the expression levels of IL-6 in the rat cortex 1, 4 and 7 days after TBI or sham operation. (B) The expression levels of IL- 6 were normalized to those of $\beta$-actin. The results are presented as the mean \pm standard error $(n=5)$. The expression levels of IL- 6 were significantly increased in the TBI group 1, 4 and 7 days after TBI (" $\mathrm{P}<0.05$, vs. sham group). Treatment with RSG significantly downregulated IL-6 expression 1,4 and 7 days after TBI ( ${ }^{* *} \mathrm{P}<0.05$, vs. TBI group). TBI, traumatic brain injury; RSG, rosiglitazone; IL-6, interleukin-6. 

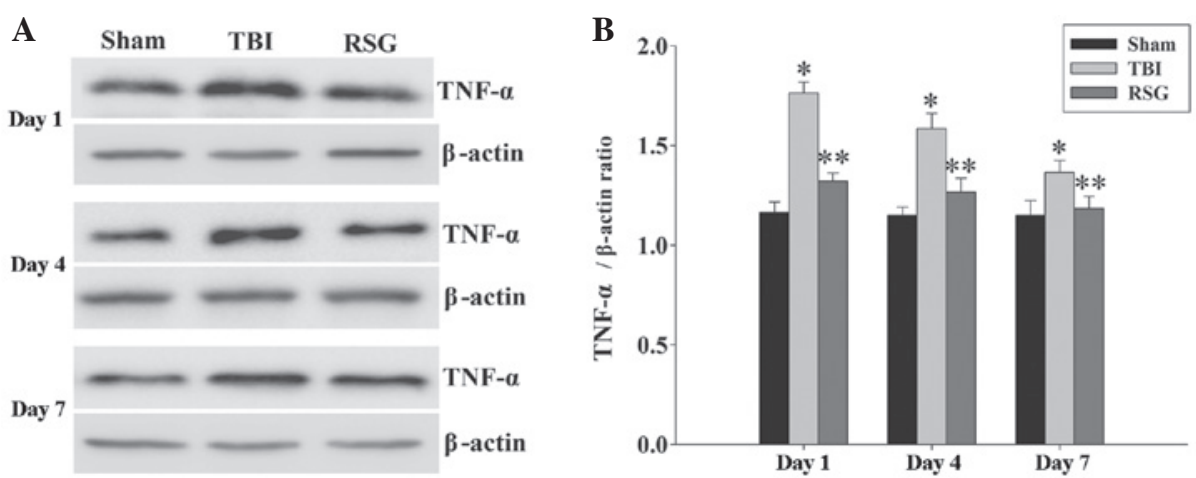

Figure 7. (A) Western blotting was used to detect the expression levels of TNF- $\alpha$ in the rat cortex 1, 4 and 7 days after TBI or sham operation. (B) The expression levels of TNF- $\alpha$ were normalzied to those of $\beta$-actin. The data are presented as the mean \pm standard error $(\mathrm{n}=5)$. The expression levels of TNF- $\alpha$ were significantly increased in the TBI group 1,4 and 7 days after TBI (" $\mathrm{P}<0.01$, vs. sham group). Treatment with RSG significantly downregulated TNF- $\alpha$ expression 1, 4 and 7 days after TBI ( ${ }^{* *} \mathrm{P}<0.05$, vs. TBI group). TBI, traumatic brain injury; RSG, rosiglitazone; TNF- $\alpha$, tumor necrosis factor.
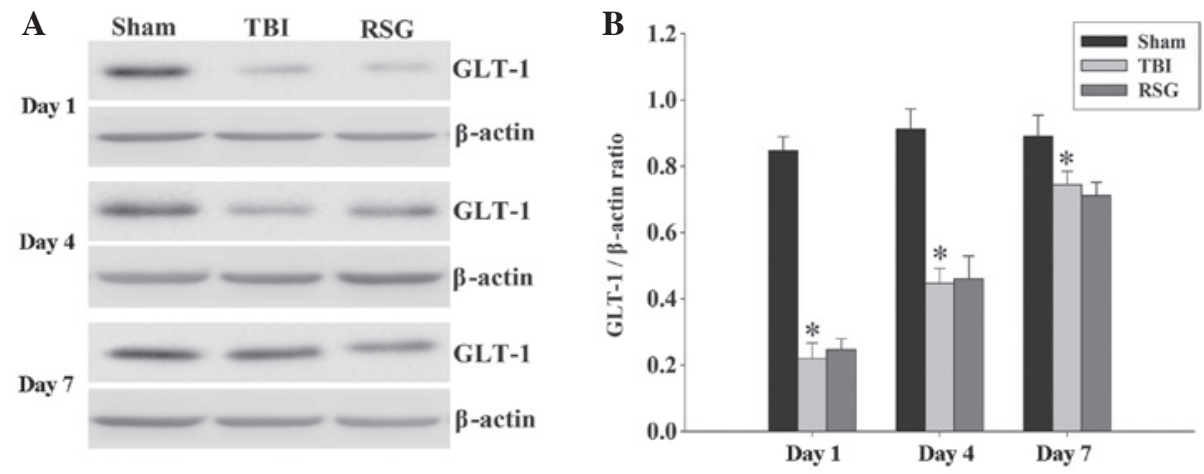

Figure 8. (A) Western blotting was used to detect the expression levels of GLT-1 in the rat cortex 1, 4 and 7 days following TBI or sham operation. (B) The expression levels of GLT-1 were normalized to those of $\beta$-actin. The data are presented as the mean \pm standard error $(n=5)$. The expression levels of GLT-1 were significantly decreased in the TBI group 1,4 and 7 days after TBI. ("P<0.01, vs. sham group). Treatment with RSG had no significant effect on the expression levels of GLT-1 1, 4 and 7 days after TBI ( $(*$ P $>0.05$, vs. TBI group). TBI, traumatic brain injury; RSG, rosiglitazone; GLT-1, glutamate transporter-1.

have demonstrated that RSG exerts neuroprotective effects in numerous acute brain injury models, including focal ischemia, spinal cord injury, and TBI (14-19). Using the CCI model of TBI, the present study confirmed these previous results, and extended these observations by providing the first demonstration, to the best of our knowledge, that post-TBI treatment with RSG exerts neuroprotective effects via the attenuation of neuronal apoptosis and autophagy in the cortex, and these neuroprotective effects were not mediated by GLT-1.

A previous study demonstrated that TBI initiates physiopathological cascades of cell death signals and induces numerous cell death pathways (20). Apoptosis is an important type of programmed cell death that occurs following TBI (8). Caspase 3, which is regarded as an effector caspase, may be activated via the amplification of extrinsic or intrinsic apoptotic signals (9). Conversely, Bcl-2 is regarded as an anti-apoptotic member of the Bcl-2 protein family, which has an important role in the regulation of caspase-dependent and caspase-independent apoptosis (10). The results of the present study demonstrated that treatment with RSG resulted in decreased protein expression levels of activated caspase 3, but increased protein expression levels of Bcl-2 in the cortex following TBI. Previous studies have suggested that treatment with RSG decreased the number of apoptotic neurons following TBI (19), results which are confirmed by the findings of the present study, which demonstrated that RSG is able to exert neuroprotective effects via attenuation of TBI-induced neuronal apoptosis.

A previous study demonstrated that autophagy is activated in damaged brain tissue samples of numerous and distinct animal brain injury models (21). Erlich et al (22) evaluated the effects of treatment with an autophagy agonist in a closed head injury model. The results indicated that treatment with rapamycin resulted in improved neurobehavioral function and increased neuronal survival in the injured region (22). Conversely, numerous studies have demonstrated that attenuation of TBI-induced neuronal autophagy improved cognitive performance and reduced histological damage $(7,23)$. Therefore, whether the role of autophagy is detrimental or beneficial following TBI remains uncertain and controversial. Notably, in the present study, treatment with RSG attenuated the TBI-induced elevated expression levels of LC3II and Beclin-1 in the brain cortex. It is therefore conceivable to hypothesize that TBI overactivates neuronal autophagy, which causes neuronal self-digestion and induces neuronal cell death, and the neuroprotection of RSG may be associated with the attenuation of TBI-induced over-activated neuronal autophagy.

TNF- $\alpha$ and IL- 6 are crucial proinflammatory cytokines involved in TBI-induced inflammatory responses (24). A previous study demonstrated that activated TNF- $\alpha$ and IL-6 
expression in the initial post-injury period is harmful, and attenuation of these cytokines may exert neuroprotective effects following TBI (25). The results of the present study demonstrated that RSG is able to downregulate the expression levels of inflammatory cytokines TNF- $\alpha$ and IL- 6 in the cortex following TBI. These results are concordant with those of previous studies, leading to the hypothesis that apoptotic and autophagic pathways may be influenced by the downregulation of inflammatory cytokines. In addition, numerous studies have confirmed the important role of glutamate-mediated excitotoxicity in the pathophysiology of TBI $(26,27)$. In the central nervous system, the activation of glutamate transporter systems, which promote glutamate uptake, is the principle mechanism by which extracellular glutamate concentrations are maintained below the level of excitotoxicity (27). Among these transporter mechanisms, GLT-1 is responsible for $\sim 90 \%$ of all glutamate transport in adult brain tissue (28). Therefore, the pharmacological modulation of GLT-1 may provide novel therapeutic applications in TBI. However, in the present study, no significant changes were observed in the protein expression levels of GLT-1 in the rats treated with RSG. The results of the present study suggested that the neuroprotective effects of RSG are not mediated by the modulation of GLT-1 expression in a rat model of TBI.

In conclusion, the present study demonstrated that treatment with RSG reduced the levels of neuronal apoptosis and autophagy, and increased the functional recovery in a rat model of TBI. Furthermore, RSG may also decrease the protein expression levels of TNF- $\alpha$ and IL- 6 , but no significant changes were observed in the protein expression levels of GLT-1 in the cortex. These results suggest that RSG may exert neuroprotective effects via the reduction of neuronal apoptosis and autophagy following experimental TBI in rats, and the mechanism underlying these neuroprotective effects may be associated with the anti-inflammatory effects of RSG.

\section{Acknowledgements}

The present study was supported by a grant from the Natural Science Foundation of Hebei Province (grant no. H2013201283)

\section{References}

1. Liu Y, Yi XC, Guo G, Long QF, Wang XA, Zhong J, Liu WP Fei Z, Wang DM and Liu J: Basic fibroblast growth factor increases the transplantation-mediated therapeutic effect of bone mesenchymal stem cells following traumatic brain injury. Mol Med Rep 9: 333-339, 2014.

2. Greve MW and Zink BJ: Pathophysiology of traumatic brain injury. Mt Sinai J Med 76: 97-104, 2009.

3. Gaetz M: The neurophysiology of brain injury. Clin Neurophysiol 115: 4-18, 2004.

4. Headrick JP, Bendall MR, Faden AI and Vink R: Dissociation of adenosine levels from bioenergetic state in experimental brain trauma: Potential role in secondary injury. J Cereb Blood Flow Metab 14: 853-861, 1994.

5. Khan M, Im YB, Shunmugavel A, Gilg AG, Dhindsa RK, Singh AK and Singh I: Administration of S-nitrosoglutathione after traumatic brain injury protects the neurovascular unit and reduces secondary injury in a rat model of controlled cortical impact. J Neuroinflammation 6: 32, 2009.

6. Cui C, Cui Y, Gao J, Sun L, Wang Y, Wang K, Li R, Tian Y, Song $\mathrm{S}$ and Cui J: Neuroprotective effect of ceftriaxone in a rat model of traumatic brain injury. Neurol Sci 35: 695-700, 2014.
7. Wang YQ, Wang L, Zhang MY, Wang T, Bao HJ, Liu WL, Dai DK, Zhang L, Chang P, Dong WW, et al: Necrostatin-1 suppresses autophagy and apoptosis in mice traumatic brain injury model. Neurochem Res 37: 1849-1858, 2012.

8. Rink A, Fung KM, Trojanowski JQ, Lee VM, Neugebauer E and McIntosh TK: Evidence of apoptotic cell death after experimental traumatic brain injury in the rat. Am J Pathol 147: 1575-1583, 1995.

9. Clark RS, Kochanek PM, Watkins SC, Chen M, Dixon CE, Seidberg NA, Melick J, Loeffert JE, Nathaniel PD, Jin KL, and Graham SH: Caspase-3 mediated neuronal death after traumatic brain injury in rats. J Neurochem 74: 740-753, 2000.

10. Graham SH, Chen J and Clark RS: Bcl-2 family gene products in cerebral ischemia and traumatic brain injury. J Neurotrauma 17: 831-841, 2000.

11. Clark RS, Bayir H, Chu CT, Alber SM, Kochanek PM and Watkins SC: Autophagy is increased in mice after traumatic brain injury and is detectable in human brain after trauma and critical illness. Autophagy 4: 88-90, 2008.

12. Cao Y and Klionsky DJ: Physiological functions of Atg6/Beclin 1: A unique autophagy-related protein. Cell Res 17: 839-849, 2007.

13. Mohanty P, Aljada A, Ghanim H, Hofmeyer D, Tripathy D, Syed T, Al-Haddad W, Dhindsa S and Dandona P: Evidence for a potent antiinflammatory effect of rosiglitazone. J Clin Endocrinol Metab 89: 2728-2735, 2004.

14. Risner ME, Saunders AM, Altman JF, Ormandy GC, Craft S, Foley IM, Zvartau-Hind ME, Hosford DA and Roses AD; Rosiglitazone in Alzheimer's Disease Study Group: Efficacy of rosiglitazone in a genetically defined population with mild-to-moderate Alzheimer's disease. Pharmacogenomics J 6: 246-254, 2006.

15. Schütz B, Reimann J, Dumitrescu-Ozimek L, Kappes-Horn K, Landreth GE, Schürmann B, Zimmer A and Heneka MT: The oral antidiabetic pioglitazone protects from neurodegeneration and amyotrophic lateral sclerosis-like symptoms in superoxide dismutase-G93A transgenic mice. J Neurosci 25: 7805-7812, 2005.

16. Chaturvedi RK and Beal MF: PPAR: A therapeutic target in Parkinson's disease. J Neurochem 106: 506-518, 2008.

17. Luo Y, Yin W, Signore AP, Zhang F, Hong Z, Wang S, Graham SH and Chen J: Neuroprotection against focal ischemic brain injury by the peroxisome proliferator-activated receptor-gamma agonist rosiglitazone. J Neurochem 97: 435-448, 2006.

18. Zhang Q, Hu W, Meng B and Tang T: PPAR $\gamma$ agonist rosiglitazone is neuroprotective after traumatic spinal cord injury via anti-inflammatory in adult rats. Neurol Res 32: 852-859, 2010.

19. Yi JH,Park SW, Brooks N, Lang BT and Vemuganti R: PPARgamma agonist rosiglitazone is neuroprotective after traumatic brain injury via anti-inflammatory and anti-oxidative mechanisms. Brain Res 1244: 164-172, 2008.

20. Stoica BA and Faden AI: Cell death mechanisms and modulation in traumatic brain injury. Neurotherapeutics 7: 3-12, 2010.

21. Xu M and Zhang HL: Death and survival of neuronal and astrocytic cells in ischemic brain injury: A role of autophagy. Acta Pharmacol Sin 32: 1089-1099, 2011.

22. Erlich S, Alexandrovich A, Shohami E and Pinkas-Kramarski R: Rapamycin is a neuroprotective treatment for traumatic brain injury. Neurobiol Dis 26: 86-93, 2007.

23. Lai Y, Hickey RW, Chen Y, Bayir H, Sullivan ML, Chu CT, Kochanek PM, Dixon CE, Jenkins LW, Graham SH, et al: Autophagy is increased after traumatic brain injury in mice and is partially inhibited by the antioxidant gamma-glutamylcysteinyl ethyl ester. J Cereb Blood Flow Metab 28: 540-550, 2008.

24. Csuka E, Morganti-Kossmann MC, Lenzlinger PM, Joller H, Trentz $\mathrm{O}$ and Kossmann T: IL-10 levels in cerebrospinal fluid and serum of patients with severe traumatic brain injury: Relationship to IL-6, TNF-alpha, TGF-beta 1 and blood-brain barrier function. J Neuroimmunol 101: 211-221, 1999.

25. He J, Evans CO, Hoffman SW, Oyesiku NM and Stein DG: Progesterone and allopregnanolone reduce inflammatory cytokines after traumatic brain injury. Exp Neurol 189: 404-412, 2004.

26. Palmer AM, Marion DW, Botscheller ML, Swedlow PE, Styren SD and DeKosky ST: Traumatic brain injury-induced excitotoxicity assessed in a controlled cortical impact model. J Neurochem 61: 2015-2024, 1993.

27. Yi JH and Hazell AS: Excitotoxic mechanisms and the role of astrocytic glutamate transporters in traumatic brain injury. Neurochem Int 48: 394-403, 2006.

28. Rao VL, Başkaya MK, Doğan A, Rothstein JD and Dempsey RJ: Traumatic brain injury down-regulates glial glutamate transporter (GLT-1 and GLAST) proteins in rat brain. J Neurochem 70: 2020-2027, 1998. 